\title{
UHRF1 depletion suppresses growth of gallbladder cancer cells through induction of apoptosis and cell cycle arrest
}

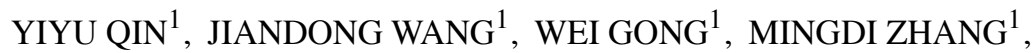 \\ ZHAOHUI TANG ${ }^{1}$, JUN ZHANG ${ }^{2}$ and ZHIWEI QUAN ${ }^{1}$ \\ ${ }^{1}$ Department of General Surgery and ${ }^{2}$ Ministry of Education-Shanghai Key Laboratory of Children's Environmental Health, \\ Xinhua Hospital Affiliated to Shanghai Jiaotong University School of Medicine, Shanghai 200092, P.R. China
}

Received December 28, 2013; Accepted February 21, 2014

DOI: $10.3892 /$ or.2014.3145

\begin{abstract}
Ubiquitin-like containing PHD and RING finger domains 1 (UHRF1), overexpressed in various human malignancies, functions as an important regulator in cell proliferation and epigenetic regulation. Depletion of UHRF1 has shown potential antitumor activities in several types of cancer. However, the role of UHRF1 in gallbladder cancer (GBC) has not been investigated. RT-PCR, western blotting and immunohistochemistry were performed to examine UHRF1 expression at mRNA and protein levels in GBC tissues and cell lines. UHRF1 siRNA and UHRF1 shRNA were used to deplete the expression of UHRF1. The results showed that UHRF1 was overexpressed in GBC and its expression correlated with advanced TNM stage and presence of lymph node metastasis. UHRF1 depletion in GBC-SD and NOZ cells markedly inhibited proliferation, migration in vitro and the ability of these cells to form tumors in vivo. UHRF1 depletion upregulated the expression of PML and triggered extrinsic and intrinsic apoptotic pathways by promoting the expression of FasL/FADD, bax, cytosolic cytochrome $c$, cleaved caspase- $8,-9$ and -3 and cleaved PRAP and by suppressing bcl-2 expression in GBC-SD and NOZ cells. In addition, UHRF1 depletion induced cell cycle arrest at G1/S transition by inducing p21 in a p53-independent manner in GBC-SD and NOZ cells. Our findings suggest that UHRF1 is involved in the
\end{abstract}

Correspondence to: Professor Zhiwei Quan, Department of General Surgery, Xinhua Hospital Affiliated to Shanghai Jiaotong University School of Medicine, 1665 Kongjiang Road, Shanghai 200092, P.R. China

E-mail: zhiwquan@163.com

Professor Jun Zhang, Ministry of Education-Shanghai Key Laboratory of Children's Environmental Health, Xinhua Hospital Affiliated to Shanghai Jiaotong University School of Medicine, 1665 Kongjiang Road, Shanghai 200092, P.R. China

E-mail: zhangjunjim@gmail.com

Key words: UHRF1, gallbladder cancer, PML, apoptosis, cell cycle proliferation and migration of GBC cells and may serve as a biomarker or even a therapeutic target for GBC.

\section{Introduction}

Gallbladder cancer (GBC) represents the most frequent and aggressive type among biliary tract malignancies. Although recent advances have been made in the diagnosis and treatment, GBC has a poor overall prognosis with a 5-year survival rate $<10 \%$ (1). Currently, radical resection remains the mainstay of treatment for GBC. However, due to lacking typical symptoms and specific biomarkers, most GBC patients are diagnosed at advanced stages with unresectable tumors. Less than $30 \%$ of GBC patients are considered surgical candidates (2). Moreover, effective adjuvant treatments including chemotherapy and radiotherapy are not well developed. Therefore, there is an urgent need to develop novel and effective therapy regimens for GBC patients. To achieve this, it is imperative to have a better understanding of the molecular biology and carcinogenic mechanisms underlying the development and progression of GBC.

Similar to other types of cancer, the development and progression of GBC are facilitated by the epigenetic silencing of tumor suppressor genes (3). Ubiquitin-like containing PHD and RING finger domains 1 (UHRF1) functions as an important regulator in cell proliferation and epigenetic regulation belonging to the RING-finger type E3 ubiquitin ligase subfamily. As a potential oncogenic factor, UHRF1 contributes to silencing of tumor suppressor genes by recruiting DNA methyltransferase 1 (DNMT1) to their hemimethylated promoters $(4,5)$. The expression of UHRF1 is cell cycle-regulated in normal cells, but stably high throughout cell cycle in numerous cancer cell lines including breast, colorectal, bladder, lung, prostate, esophageal and laryngeal cancer (6-12). Downregulation of UHRF1 expression showed antitumor activities in cancer cells $(13,14)$. Therefore, UHRF1 is likely to be a novel target of pivotal interest for developing new anticancer drugs.

However, the role of UHRF1 in GBC remains unclear. In the present study, UHRF1 expression in GBC tissues and cell lines was examined. The effects of UHRF1 depletion on proliferation, cell cycle, apoptosis and migration of GBC cell lines in vitro and in vivo were also investigated. 


\section{Materials and methods}

Patients and tissue samples. Tumor samples from 38 patients with GBC (12 men and 26 women; age range, 51-73 years; mean age, $62.4 \pm 5.2$ years) treated with radical cholecystectomy (without prior radiotherapy or chemotherapy) and corresponding non-tumor samples from 12 of these GBC cases were collected between 2011 and 2013 at the Department of General Surgery, Xinhua Hospital, School of Medicine, Shanghai Jiaotong University. The tumors were staged according to the TNM staging system (AJCC, 7th edition, 2010); 4 were stage I; 10 stage II; 15 stage III and 9 stage IV. The histological diagnosis of all GBC cases was confirmed by two pathologists. The present study was approved by the Ethics Committee of Xinhua Hospital, School of Medicine, Shanghai Jiaotong University and all patients provided informed consent.

Immunohistochemistry. Tissue sections $(4 \mu \mathrm{m})$ were cut from paraffin-embedded human GBC tissues and xenograft tumors, deparaffinized within xylene and rehydrated within a graded series of ethanol solutions. To block endogenous peroxidase activity, the sections were treated for $30 \mathrm{~min}$ with methanol containing $1 \%$ hydrogen peroxide. The sections were incubated with anti-UHRF1 mouse monoclonal antibody (1:50 dilution) followed by incubation with secondary antibody (1:1,000 dilution) (both from Santa Cruz Biotechnology, Inc., Santa Cruz, CA, USA). The score on the intensity of staining was graded by using a 3 -scale system $(0$, negative; 1 , weak; 2 , moderate; and 3 , strong).

Cell cultures and transfection. Human GBC cell line (GBC-SD) was obtained from the Cell Bank of the Chinese Academy of Sciences, Shanghai, China; human GBC cell line (SGC996) was obtained from the Academy of Life Science, Tongji University, Shanghai, China; human GBC cell lines (NOZ and OCUG-1) were obtained from the Health Science Research Resources Bank, Osaka, Japan. All cell lines were maintained either in Dulbecco's modified Eagle's medium (DMEM) or RPMI (Invitrogen, Carlsbad, CA, USA) at $37^{\circ} \mathrm{C}$ in a humidified 5\% $\mathrm{CO}_{2}$ incubator. Both media were supplemented with $10 \%$ fetal bovine serum (FBS) and $1 \%$ penicillin/streptomycin (both from Invitrogen).

Cells in the exponential phase of growth were transfected with siRNA or shRNA (Santa Cruz Biotechnology, Inc.) according to the manufacturer's protocol. Briefly, cells were incubated with siRNA or shRNA transfection medium for $6 \mathrm{~h}$ at $37^{\circ} \mathrm{C}$ in a $\mathrm{CO}_{2}$ incubator. Then, the transfection medium was replaced with fresh growth medium containing 2 times the normal serum and antibiotics concentration. Cells were incubated for an additional $24 \mathrm{~h}$ and then cultured under conditions normally used to culture the cells. To obtain stable transfectants, UHRF1-shRNA-transfected cells were selected with puromycin $(1-2 \mu \mathrm{g} / \mathrm{ml})$ for 10 days. Stable transfectants were used to establish xenograft tumor models. At the indicated time points post transfection, transfected cells were harvested and the silencing of UHRF1 was examined using western blot assay.

$R T-P C R$. Total RNA was extracted from GBC-SD, NOZ, SGC996 and OCUG-1 cells using TRIzol reagent kit
(Gibco-BRL, Gaithersburg, MD, USA). RNA extracts were reverse-transcribed using a Takara RNA PCR kit (Takara Bio, Inc., Dalian, China) and the obtained cDNA was subjected to PCR. The sequences of the primers for UHRF1 were: forward, gtcgagatctttccggcaac and reverse, tagatgccatcgtagcggtt.

Western blot analysis. Forty-eight hours after transfection with UHRF1 siRNA, total proteins were extracted from GBC cells and the protein content was measured using the Bradford assay. The amount of cytosolic cytochrome $c$ was determined using cytosolic extract. Mitochondrial and cytosolic fractions were extracted from cultured cells by Mitochondria/Cytosol Fractionation kit (Abcam, Cambridge, UK). Forty micrograms of proteins were separated by 10-12\% SDS-PAGE and electrotransferred to PVDF membranes (Millipore, Billerica, MA, USA). The blot was blocked with 5\% non-fat dry milk, and incubated with corresponding primary antibodies, followed by incubation with corresponding secondary antibodies (both from Santa Cruz Biotechnology, Inc.). The signals were detected by enhanced chemiluminescence (ECL) assay and visualized using X-OMAT Blue film.

Cell proliferation and clonogenic assays. Cell proliferation was determined by the water soluble tetrazolium (WST)-1 method using WST-1 cell proliferation and cytotoxicity assay kit (Beyotime, Shanghai, China). Briefly, $24 \mathrm{~h}$ after transfection with UHRF1 siRNA, GBC-SD and NOZ cells were seeded at a density of $5 \times 10^{3} /$ well into 96 -well plates and cultured for $24,48,72$ or $96 \mathrm{~h}$. The cells were then incubated with WST-1 reagent for $2 \mathrm{~h}$ at $37^{\circ} \mathrm{C}$. The optical density was measured at $450 \mathrm{~nm}$ with an automated microplate reader (Bio-Rad Model 550; Bio-Rad, Hercules, CA, USA).

The clonogenic assay was performed by plating UHRF1siRNA-transfected cells $\left(1 \times 10^{3} /\right.$ well $)$ in 6 -well plates. Transfected cells were further cultured in the growth media at $37^{\circ} \mathrm{C}$ and allowed to form colonies for 10-14 days. Colonies were stained with crystal violet and counted using an automated colony counter system (Alpha Innotech, San Leandro, CA, USA).

Cell cycle analysis. Forty-eight hours after transfection with UHRF1 siRNA, cells were harvested and fixed with $70 \%$ methanol overnight at $4^{\circ} \mathrm{C}$. Fixed cells were stained with RNase A and propidium iodide (PI) followed by flow cytometric analysis (Beckman Coulter, Brea, CA, USA). The percentage of cells in each phase of the cell cycle was analyzed using Cylchred version 1.0.2 software (Cardiff University, Wales, UK).

Cell apoptosis assay. The cell apoptosis was determined by Annexin V-FITC apoptosis detection kit (BD Biosciences, San Diego, CA, USA) according to the manufacturer's protocol. Briefly, $48 \mathrm{~h}$ after transfection with UHRF1 siRNA, cells were collected, washed twice with cold PBS, and resuspended in $1 \mathrm{X}$ binding buffer. Then, the cells were stained with FITC-Annexin V and PI for $15 \mathrm{~min}$ at room temperature in the dark. The percentage of apoptotic cells was determined by flow cytometry and the analysis was carried out within $1 \mathrm{~h}$.

Cell migration assay. The migration of transfected cells was measured using a Transwell chamber (Corning Inc., Corning, 
NY, USA) with $8-\mu \mathrm{m}$ pores. The UHRF1-siRNA-transfected cells with serum-free DMEM or RPMI-1640 were placed in the upper chamber compartment. The lower chambers contained DMEM or RPMI-1640 with $10 \%$ FBS as chemoattractant. After incubation for $24 \mathrm{~h}$ at $37^{\circ} \mathrm{C}$, cells on the lower side of the insert filter were fixed by $4 \%$ paraformaldehyde for $30 \mathrm{~min}$ and then stained with crystal violet for $20 \mathrm{~min}$.

Tumor xenograft experiments. All animal experiments were approved by the Ethics Committee of Xinhua Hospital, Shanghai Jiaotong University. Four-week-old female BALB/c nude mice were obtained from Shanghai Laboratory Animal Center of the Chinese Academy of Sciences and housed under specific pathogen-free (SPF) conditions. GBC-SD and NOZ cells which were stably transfected with UHRF1 shRNA or control shRNA were used to establish subcutaneous tumor models. Briefly, the transfected GBC-SD or NOZ cells were injected subcutaneously into the right flank region of nude mice with a concentration of $1 \times 10^{7}$ cells $/ 0.1 \mathrm{ml}$. Six weeks later, the mice were sacrificed. The xenograft tumors were collected for further investigation. The tumor volumes in each group were evaluated using the following formula: Volume $=$ length $\mathrm{x}$ width $^{2} / 2$.

TUNEL assay. Apoptosis in xenograft tumors was assessed by terminal deoxynucleotidyl transferase-mediated dUTP nick end labeling (TUNEL) staining using FragEL DNA Fragmentation Detection kit (Merck, Darmstadt, Germany). Briefly, after deparaffinization, rehydration and permeabilization, the endogenous peroxidase activity was eliminated. Subsequently, the tissue sections were incubated with TUNEL reaction mixture, and then counterstained with methyl green. A dark brown 3,3'-diaminobenzidine tetrachloride (DAB) signal indicated positive-TUNEL staining (apoptotic cell) while shades of blue to green indicated viable cells. The number of apoptotic cells was counted under a microscope and the results were presented as means \pm SD.

Statistical analysis. Statistical analysis was performed with SPSS 19.0 (SPSS Inc., Chicago, IL, USA). The numerical data are expressed as means $\pm \mathrm{SD}$. The differences between groups were evaluated using Student's t-test or one-way ANOVA or Chi-square test. $\mathrm{P}<0.05$ was considered to indicate a statistically significant difference.

\section{Results}

The expression of UHRF1 in GBC tissues and cell lines. Immunohistochemical staining showed that positive-staining for UHRF1 was found abundantly in the nuclei of cancer cells $(63.2 \%, 24 / 38)$ and occasionally in the nuclei of normal epithelial cells $(33.3 \%, 3 / 12)(\mathrm{P}<0.05$; Fig. 1A-F). The score of UHRF1-positive immunostaining in GBC tissues was much higher than that in normal tissues $(\mathrm{P}<0.05$; Fig. $1 \mathrm{G})$. Additionally, the expression of UHRF1 correlated with advanced stage and lymph node metastasis $(\mathrm{P}<0.05$; Table I).

UHRF1 mRNA and protein were detected in GBC-SD, NOZ, SGC996 and OCUG-1 cell lines (Fig. 1H). Since GBC-SD and NOZ cell lines have higher levels of UHRF1 mRNA and protein than the other two cell lines (Fig. 1I and J),
Table I. Association between UHRF1 expression and clinicopathological characteristics of gallbladder cancer.

\begin{tabular}{lrcr}
\hline & \multicolumn{3}{c}{ UHRF1 expression } \\
\cline { 2 - 4 } $\begin{array}{l}\text { Clinicopathological } \\
\text { characteristics }\end{array}$ & Total & Positive no. (\%) & P-value \\
\hline Gender & & & $>0.05$ \\
Male & 12 & $7(58.3)$ & \\
Female & 26 & $17(65.4)$ & \\
Age (years) & & & $>0.05$ \\
$\leq 60$ & 10 & $5(50.0)$ & \\
$>60$ & 28 & $19(67.9)$ & \\
TNM stage & & & \\
I & 4 & $1(25.0)$ & \\
II & 10 & $4(40.0)$ & \\
III & 15 & $11(73.3)$ & \\
IV & 9 & $8(88.9)$ & \\
Lymph node metastasis & & & \\
Yes & 22 & $18(81.2)$ & \\
No & 16 & $6(37.5)$ & \\
Presence of gallstones & & & \\
Yes & 18 & $12(66.7)$ & \\
No & 20 & $12(60.0)$ & \\
\hline
\end{tabular}

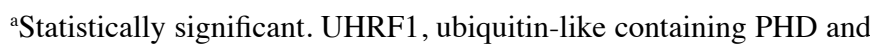
RING finger domains 1 .

they were chosen for the subsequent studies. As expected, western blot analysis showed the expression of UHRF1 protein was decreased in GBC-SD and NOZ cells after transfection with UHRF1 siRNA or UHRF1 shRNA (Fig. 1K and L).

UHRF1 depletion inhibits the growth of GBC-SD and NOZ cell lines in vitro and in vivo. WST-1 assay showed that transfection with UHRF1 siRNA markedly inhibited proliferation of GBC-SD and NOZ cells in a time-dependent manner $(\mathrm{P}<0.05$ from 24 to $96 \mathrm{~h})$ in comparison with untransfected and control-siRNA-transfected cells (Fig. 2A and B).

To evaluate the effects of UHRF1 depletion on more long-term cell proliferation, colony formation assay was also performed (Fig. 2C). The colony numbers were significantly reduced in GBC-SD and NOZ cells transfected with UHRF1 siRNA in comparison with those in GBC-SD and NOZ cells untransfected or transfected with control siRNA $(\mathrm{P}<0.05$; Fig. 2D).

All animals injected with GBC-SD or NOZ cells transfected with control shRNA developed visible tumors within the first 3 weeks of the experiment, and these xenograft tumors grew progressively. However, none of the animals that received GBC-SD or NOZ cells transfected with UHRF1 shRNA developed visible tumors within the first 3 weeks. Moreover, one of the animals that was injected with UHRF1shRNA-transfected NOZ cells did not develop tumors during 6 weeks of observation (Fig. 2E and F). The volume of the xenograft tumors formed by UHRF1-shRNA-transfected 

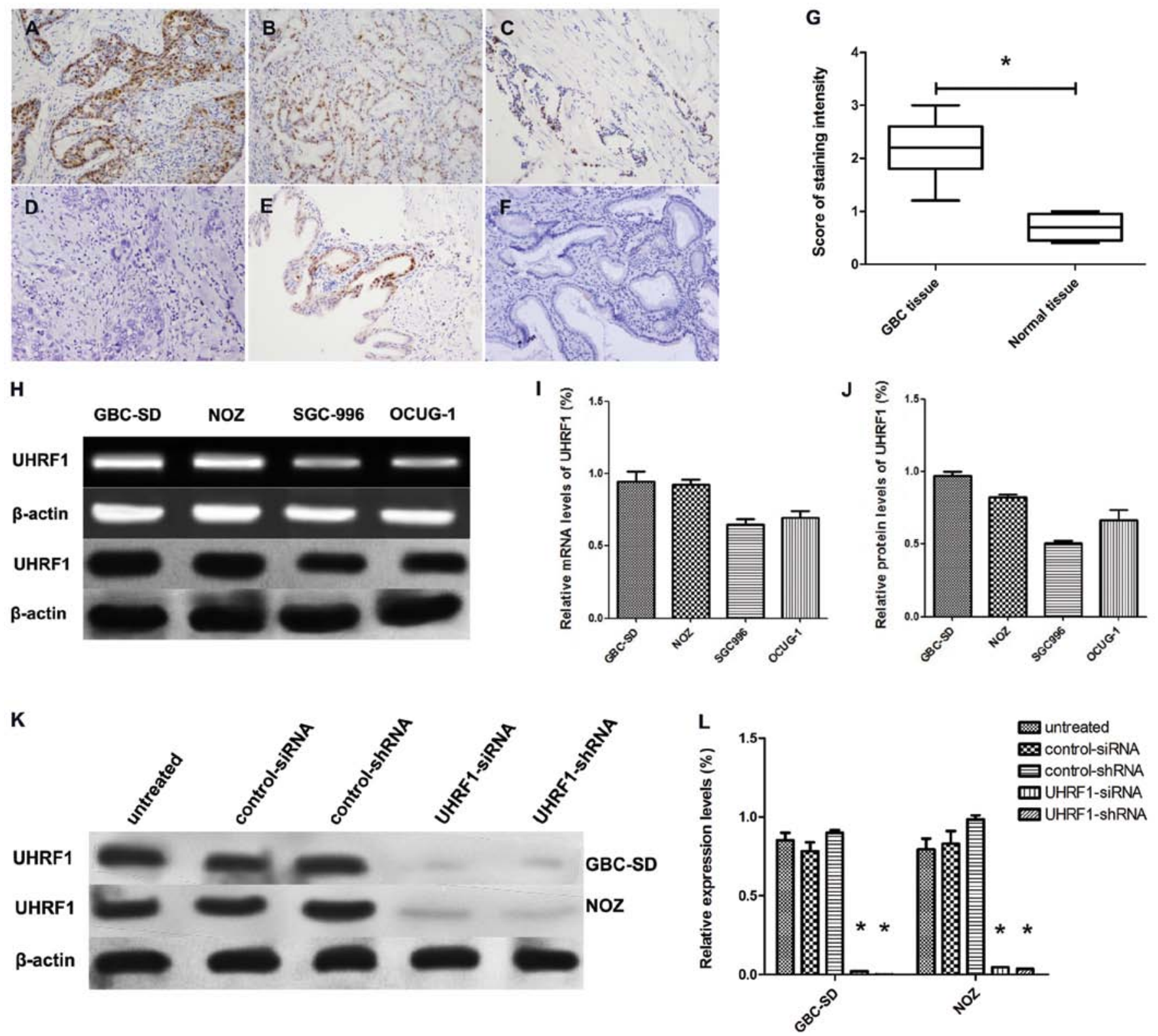

Figure 1. The expression of UHRF1 in GBC tissues and cell lines. Immunohistochemical staining (x200) showed that UHRF1 protein was located in nuclei of tumor cells and normal epithelial cells. (A) Strong staining intensity of UHRF1 in tumor cells. (B) Moderate staining intensity of UHRF1 in tumor cells. (C) Weak staining intensity of UHRF1 in tumor cells. (D) Negative staining of UHRF1 in tumor cells. (E) Weak staining intensity of UHRF1 in normal epithelial cells. (F) Negative staining of UHRF1 in normal epithelial cells. (G) The score of staining intensity of GBC tissues was much higher than that of the control group $(\mathrm{P}<0.05)$. (H-J) The mRNA and protein levels of UHRF1 in GBC-SD, NOZ, SGC996 and OCUG-1 cell lines. (K and L) UHRF1 siRNA and UHRF1 shRNA significantly decreased the levels of UHRF1 protein in GBC-SD and NOZ cells $(\mathrm{P}<0.05)$. UHRF1, ubiquitin-like containing PHD and RING finger domains 1; GBC, gallbladder cancer.

cells was significantly smaller than that of the control groups (P<0.05; Fig. 2G).

UHRF1 depletion correlates with high apoptosis in GBC cell lines and xenograft tumors. The effects of UHRF1 depletion on apoptosis in vitro and in vivo were determined using Annexin V/PI assay and TUNEL assay, respectively. Annexin V/PI assay showed that UHRF1 siRNA triggered apoptosis (early apoptosis plus late apoptosis) in GBC-SD cells and NOZ cells (Fig. 3A). The proportions of apoptotic GBC-SD cells and NOZ cells after transfection with UHRF1 siRNA were much higher than those of apoptotic GBC-SD cells and NOZ cells untransfected or transfected with control siRNA ( $\mathrm{P}<0.05$; Fig. 3B). TUNEL assay showed that stable transfection with UHRF1 shRNA increased the percentage of TUNEL-positive cells both in GBC-SD and NOZ cells in vivo (Fig. 3C). The mean numbers of apoptotic GBC-SD and NOZ cells transfected with UHRF1 shRNA/field were $17.7 \pm 2.5$ and $24.7 \pm 4.7$, both of which were much more than those of the control groups $(\mathrm{P}<0.05$; Fig. 3D).

UHRF1 depletion activates extrinsic and intrinsic apoptotic pathways in GBC-SD and NOZ cells in vitro. The expressions of a series of pro-apoptotic and anti-apoptotic proteins that 
A

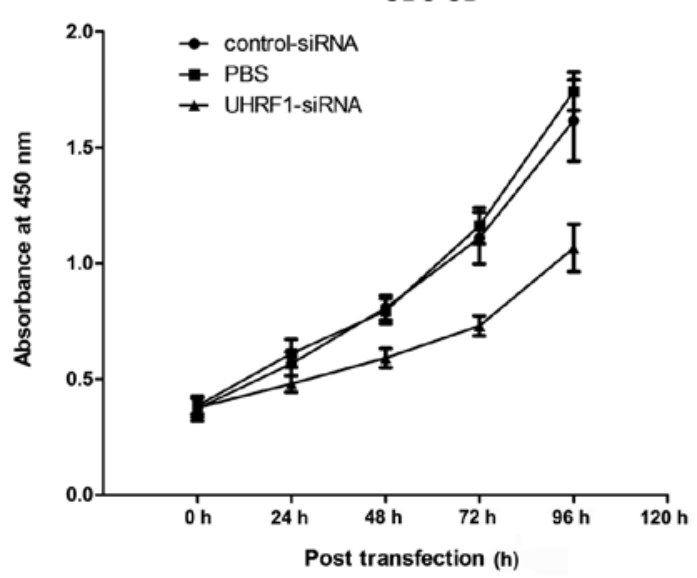

B

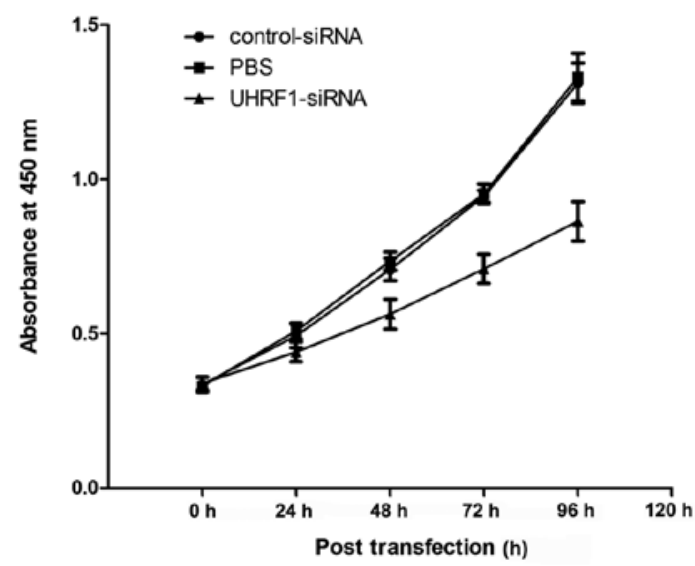

C

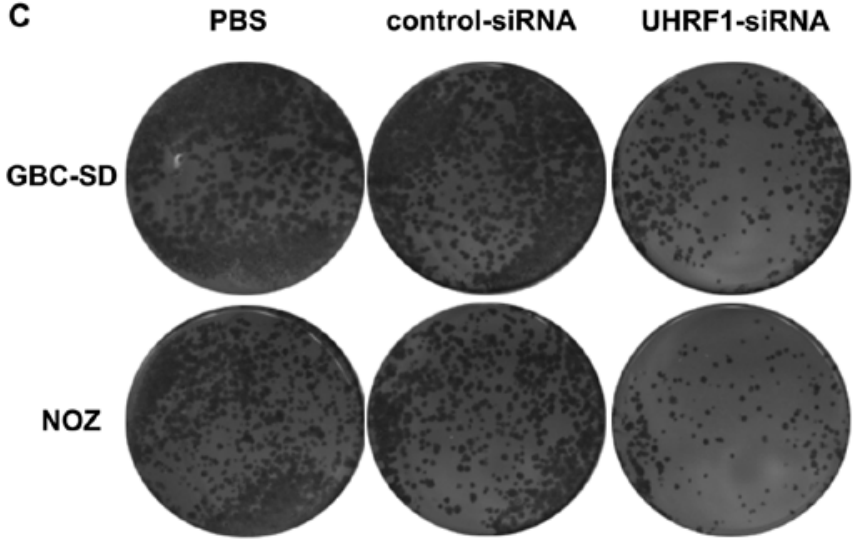

E

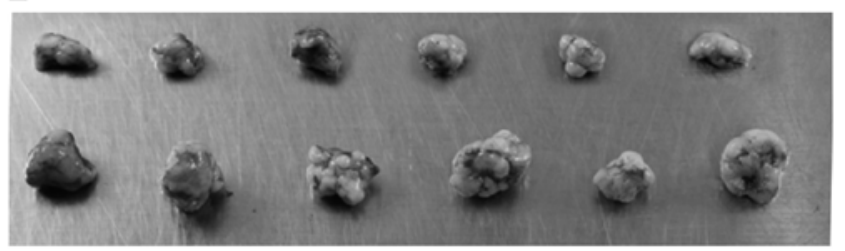

F

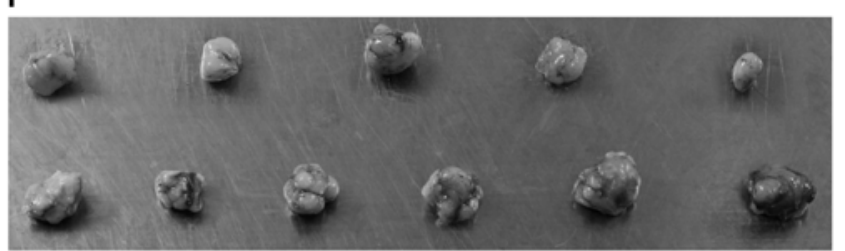

D
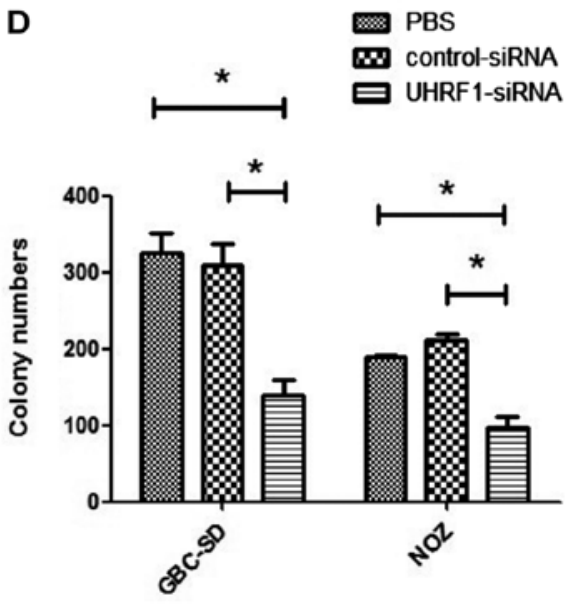

G

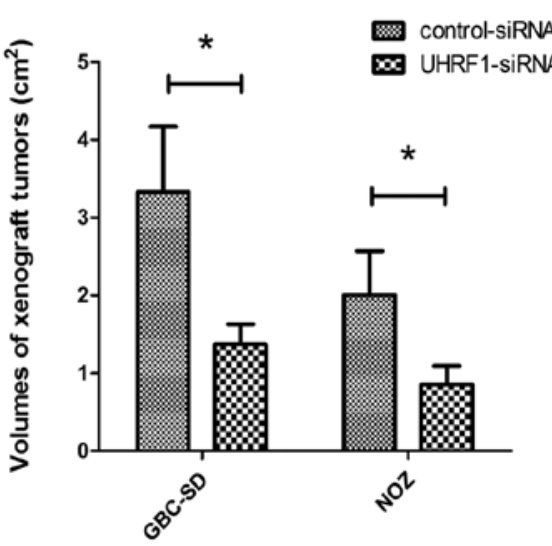

Figure 2. Effects of UHRF1 depletion on GBC cell line growth in vitro and in vivo. Growth inhibition of GBC-SD and NOZ cells induced by UHRF1 siRNA began at $24 \mathrm{~h}$ and reached a peak at $96 \mathrm{~h}(\mathrm{P}<0.05$ from 24 to $96 \mathrm{~h})$. (A and B) No significant difference in growth inhibition was observed between control-siRNA-treated and PBS-treated group. (C) Colony formation by GBC-SD and NOZ cells after transfection with UHRF1 siRNA or control siRNA. (D) Colony numbers of GBC-SD and NOZ cells were significantly decreased after transfection with UHRF1 siRNA (P<0.05). (E) Xenograft tumor in mice injected with GBC-SD cells (first row, UHRF1-shRNA; second row, control-shRNA). (F) Xenograft tumor in mice injected with NOZ cells (first row, UHRF1shRNA; second row, control-shRNA). (G) The volumes of xenograft tumors formed by UHRF1-shRNA-transfected cells were much smaller than those of the control group $(\mathrm{P}<0.05)$. UHRF1, ubiquitin-like containing PHD and RING finger domains 1; GBC, gallbladder cancer.

may participate in the process of apoptosis induced by UHRF1 depletion were examined using western blot assay. As shown in Fig. 4, the pro-caspase- $3,-8$ and -9 and bcl-2 were decreased while the cleaved caspase- $3,-8$ and -9 and PARP, Bax, cytosolic cytochrome $c$, FasL and FADD were increased after transfection with UHRF1 siRNA. In addition, the expression 
Table II. Cell cycle analysis after transfection.

Cell cycle distribution (G1, S, G2)

\begin{tabular}{llccr}
\cline { 3 - 4 } Cell line & Transfection & G1 phase $(\%)$ & S phase (\%) & G2 phase (\%) \\
\hline GBC-SD & UHRF1 siRNA & $75.5 \pm 4.5^{\mathrm{a}}$ & $14.4 \pm 2.4$ & $10.0 \pm 6.9$ \\
& Control siRNA & $55.7 \pm 2.6$ & $29.1 \pm 4.0$ & $15.2 \pm 4.2$ \\
& PBS & $57.3 \pm 4.3$ & $17.9 \pm 3.2$ & $24.9 \pm 3.3$ \\
NOZ & UHRF1 siRNA & $78.5 \pm 4.3^{\mathrm{a}}$ & $10.9 \pm 1.2$ & $8.6 \pm 1.8$ \\
& Control siRNA & $58.7 \pm 3.9$ & $22.8 \pm 3.2$ & $18.5 \pm 1.3$ \\
& PBS & $60.6 \pm 1.7$ & $22.0 \pm 2.4$ & $17.4 \pm 3.3$ \\
\hline
\end{tabular}

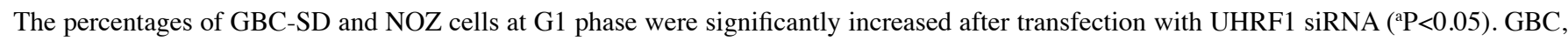
gallbladder cancer; UHRF1, ubiquitin-like containing PHD and RING finger domains 1; PBS, phosphate-buffered saline.

A
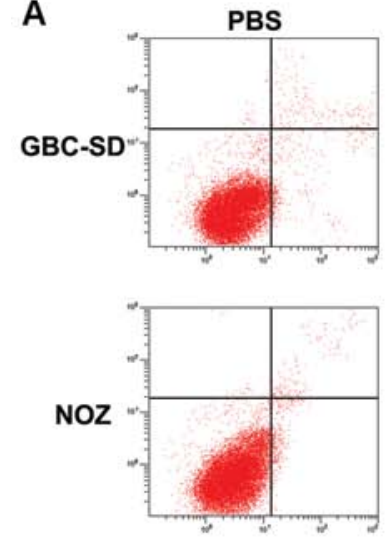

control-siRNA
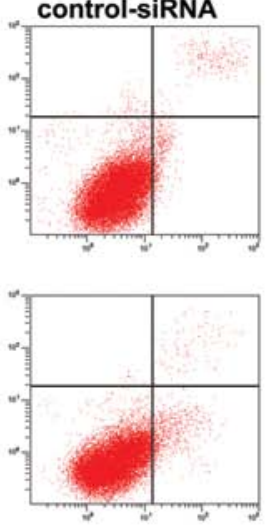
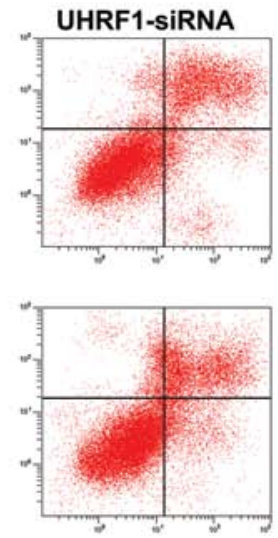

UHRF1-shRNA

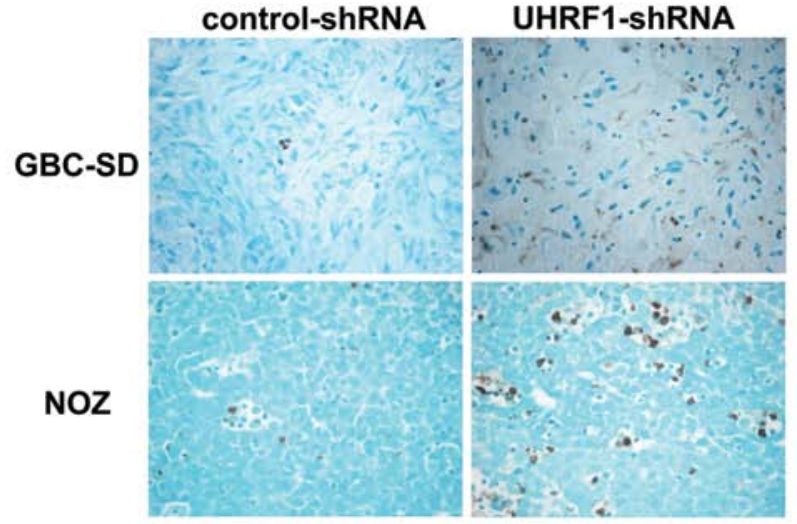

B

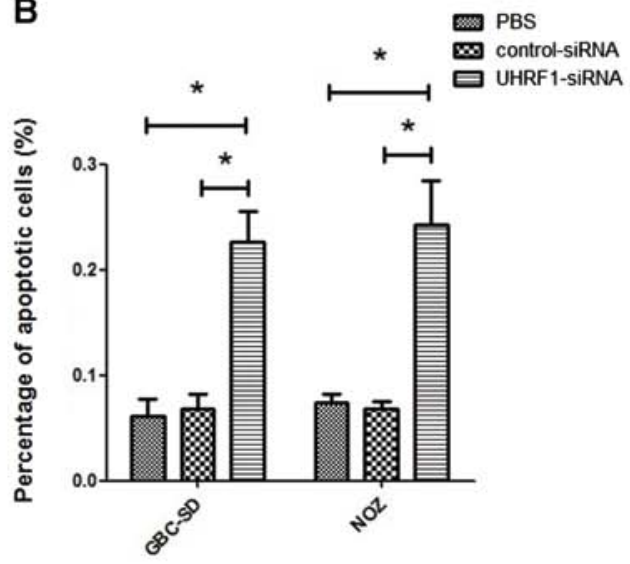

D

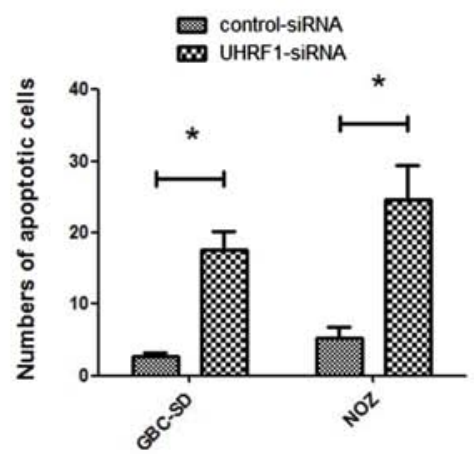

Figure 3. Effects of UHRF1 depletion on induction of apoptosis in GBC-SD and NOZ cell lines. (A) Cells that stain negative for both Annexin V and PI are viable cells (lower left). Cells that stain positive for Annexin V and negative for PI are undergoing early apoptosis (lower right). Cells that stain positive for both Annexin V and PI are either at the end stage of apoptosis or undergoing necrosis (upper right). (B) The percentages of apoptotic GBC-SD and NOZ cells (undergoing early apoptosis, late apoptosis and necrosis) were significantly increased after transfection with UHRF1 siRNA (P<0.05). (C) Apoptotic cells were counted under high-power fields (x400). (D) UHRF1 shRNA significantly promoted apoptosis of GBC-SD and NOZ cells in vivo (P<0.05). UHRF1, ubiquitin-like containing PHD and RING finger domains 1; GBC, gallbladder cancer.

levels of PML and p21 were increased after transfection with UHRF1 siRNA, while the expression of p53 was not altered in the same conditions.

UHRF1 depletion arrests GBC-SD and NOZ cells at G1 phase. Transfection with UHRF1 siRNA induced an obvious cell cycle arrest at G1 phase in GBC-SD and NOZ cells $(\mathrm{P}<0.05$; Table II), and the percentages of cells at S and G2 phase were correspondingly decreased.

UHRF1 depletion reduces the migration of GBC-SD and NOZ cells in vitro. The number of migratory cells stained 
A

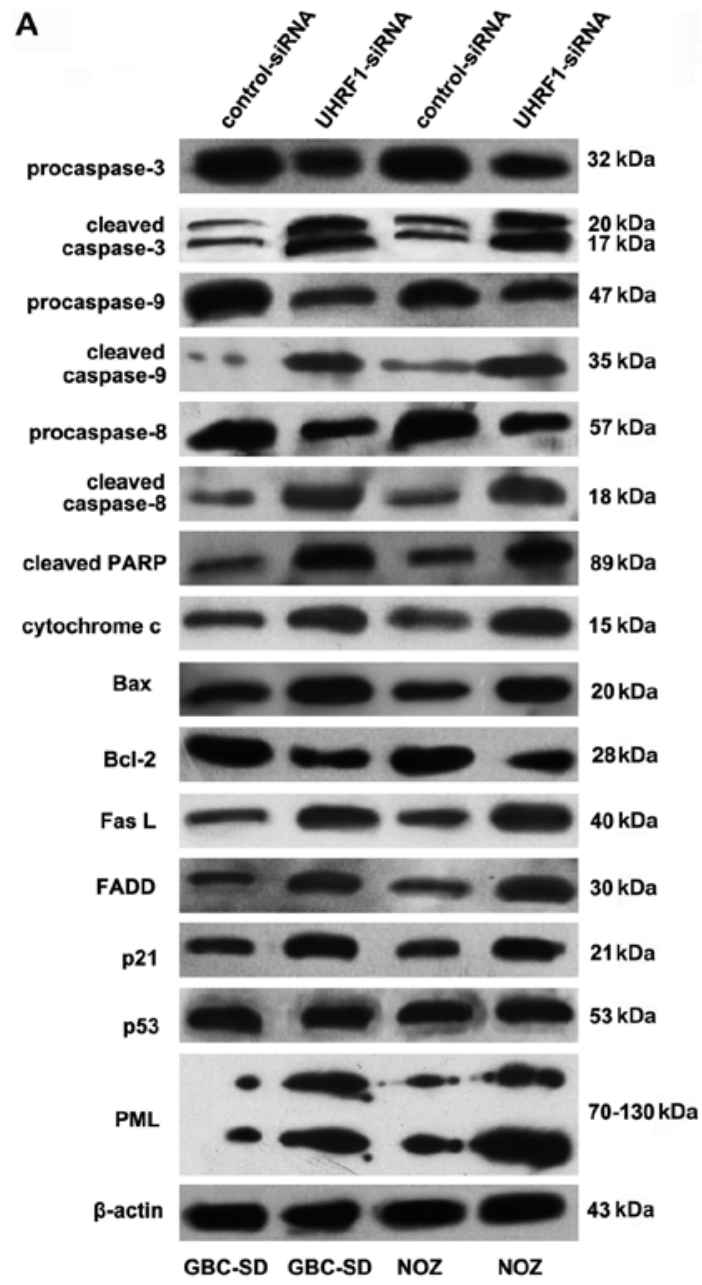

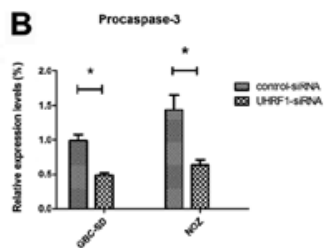
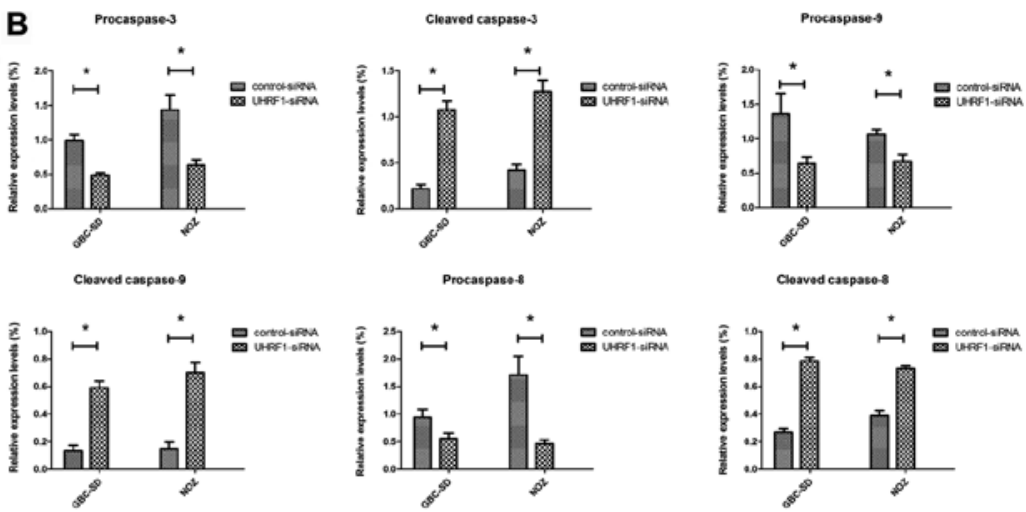

Cleaved PARP
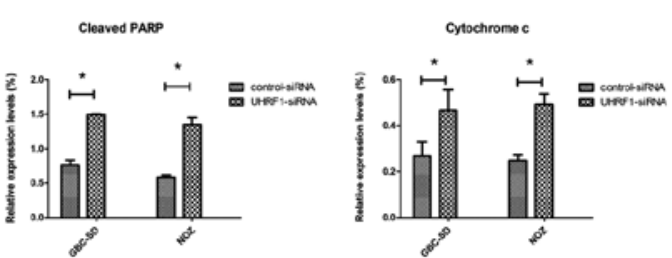

Bax

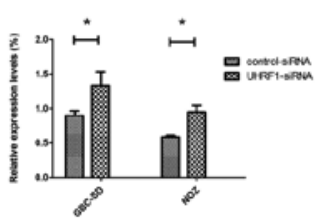

Bel-2
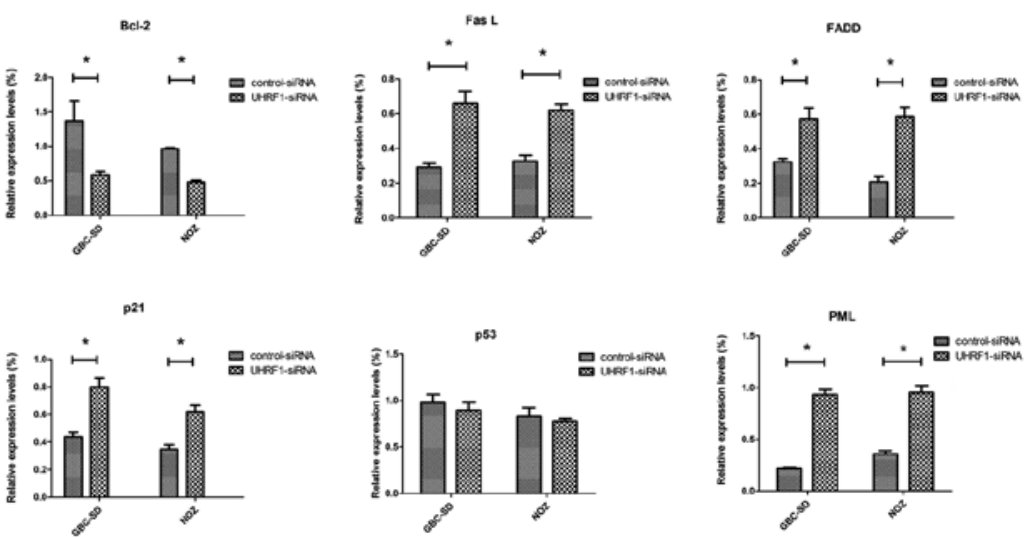

Figure 4. Effects of UHRF1 depletion on the expressions of apoptosis-related and cell cycle-related proteins. (A) The representative target bands are shown. The optical density of target bands and control bands ( $\beta$-actin) was quantified using Image-Pro Plus 6.0 software (Media Cybernetics, Silver Spring, MD, USA). The density ratios of target bands to control bands were used to determine the relative level of each protein. (B) The relative expression of each protein was calculated and shown as histogram. UHRF1, ubiquitin-like containing PHD and RING finger domains 1.

A

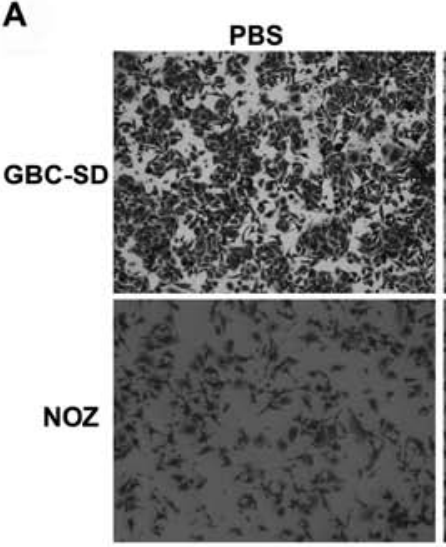

control-siRNA

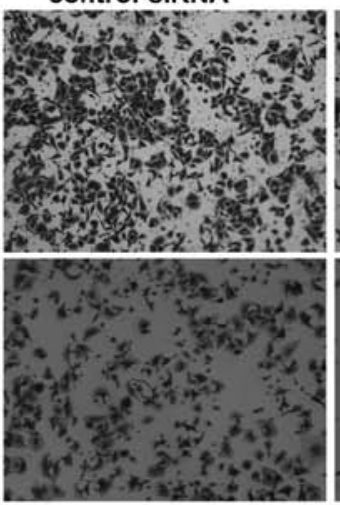

UHRF1-SIRNA

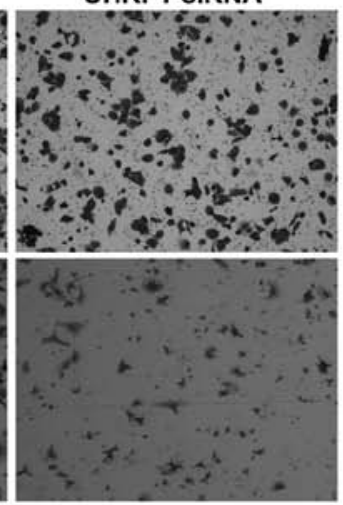

B
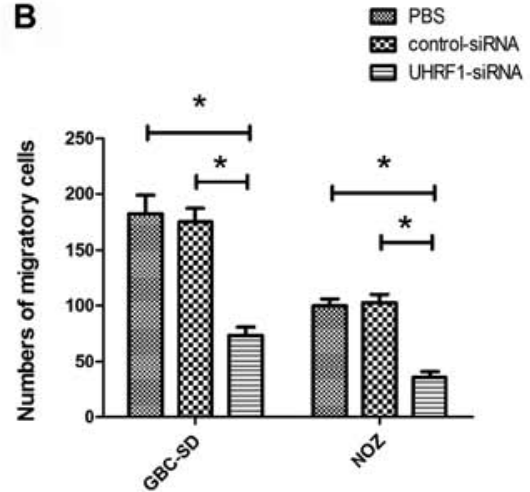

Figure 5. Effects of UHRF1 depletion on migration of GBC-SD and NOZ cells. (A) The numbers of migratory GBC-SD and NOZ cells/visual field were counted under microscopy after crystal violet staining (x100). (B) Transfection with UHRF1 siRNA significantly inhibited migration of GBC-SD and NOZ cells $(\mathrm{P}<0.05)$. UHRF1, ubiquitin-like containing PHD and RING finger domains 1; GBC, gallbladder cancer.

with crystal violet reflected their migratory capacity (Fig. 5A). Transfection with UHRF1 siRNA resulted in a significant decrease in the numbers of migratory GBC-SD and NOZ cells in comparison with the control groups $(\mathrm{P}<0.05$; Fig. 5B). 


\section{Discussion}

In the present study, the role of UHRF1 in GBC was preliminarily investigated. First, we examined the expression of UHRF1 in GBC tissues and cell lines. Consistent with other cancer tissues, immunohistochemical staining showed that UHRF1 protein was overexpressed in GBC tissues in comparison with non-tumor tissues. The positive expression of UHRF1 correlated with advanced TNM stage and lymph node metastasis, but not with age, gender and presence of gallstones. Since the expression of UHRF1 was regulated by p53, the frequent abnormality of p53 detected in GBC (15) would be an explanation for the observed UHRF1 overexpression. However, the 5-year survival rate and median survival time could not be calculated now, therefore the relationship between UHRF1 expression and prognosis was not evaluated in the present study. These observations indicate that UHRF1 may play a role in the tumorigenesis and progression of GBC.

To further explore the possible biological function of UHRF1 in GBC growth and development, UHRF1 expression was depleted in GBC-SD and NOZ cell lines using siRNA or shRNA. Our findings showed that UHRF1 depletion significantly inhibited proliferation in vitro and tumorigenicity in vivo, accompanied by cell cycle arrest and induction of apoptosis. Comparable to the results of other studies that UHRF1 depletion may cause cell cycle arrest either at G1 $(14,16)$ or G2/M phase (13), our results showed that the UHRF1 depletion induced cell cycle arrest at G1 phase in GBC cell lines. These findings indicate that UHRF1 may play a critical role during multiple phases of the cell cycle and the effects of UHRF1 depletion on cell cycle arrest may be cell-type specific.

Apoptosis induction by UHRF1 depletion has been reported in several studies, but the exact molecular mechanisms are not fully understood. As is known, caspase family members are crucial mediators during the process of apoptosis. Among them, caspase-3 is a key effector in the execution of the apoptotic program and is primarily responsible for the cleavage of poly-(ADP-ribose) polymerase (PARP) (17). The latter serves as a sensitive parameter for identification of different types of cell death and as a marker for activation of different death proteases. Caspase- 8 activation is typically associated with the extrinsic or death receptor pathway of apoptosis, whereas caspase- 9 activation following mitochondrial disruption and cytochrome $c$ release is associated with the intrinsic or mitochondrial pathway of apoptosis (18). In the present study, we found the expressions of cleaved caspase-3, -8 and -9, PARP, FasL, FADD, bax and cytosolic cytochrome $c$ were significantly increased, while the expressions of pro-caspase- $-3,-8$ and -9 and bcl- 2 were significantly decreased. These findings suggested that UHRF1 siRNA induced both extrinsic and intrinsic apoptotic pathways in GBC-SD an NOZ cells.

In addition to growth inhibition, UHRF1 siRNA also inhibited the migration of GBC-SD and NOZ cells, which indicated that UHRF1 may play a role in the migration of GBC cells. This result also supported the aforementioned finding that the overexpression of UHRF1 correlated with late TNM stage and lymph node metastasis of GBC.

UHRF1 depletion could reactivate the expressions of these tumor suppressor genes such as PML (19), which was frequently downregulated in GBC tissues (20). In the present study, we found PML expression was markedly increased after transfection with UHRF1 siRNA. Considering that PML was functionally linked to various fundamental cellular processes including caspase-dependent apoptosis in response to Fas L/Fas signals and control of cell migration $(21,22)$, we suggested that reactivation of PML by UHRF1 depletion may lead to induction of apoptosis and inhibition of migration in GBC cell lines. In addition, the expressions of two other tumor suppressor genes p53 and p21 were also examined, both of which play important roles in apoptosis and cell cycle regulation. The level of p21 protein was significantly increased, but the level of $\mathrm{p} 53$ protein which acts as a transcriptional activator of p21 was not altered, suggesting UHRF1 depletion results in an accumulation of $\mathrm{p} 21$ in a p53-independent manner. Induction of p53-independent $\mathrm{p} 21$ which was also observed in other studies $(23,24)$ may be a reasonable explanation for the G1 arrest caused by UHRF1 siRNA. These findings further indicated that UHRF1 induced p53-independent, Fas mediated apoptosis in GBC-SD and NOZ cell lines.

Taken together, we found UHRF1 was overexpressed in GBC tissues and UHRF1 depletion by siRNA or shRNA inhibited proliferation and migration in GBC cell lines. In addition, UHRF1 depletion induced cell cycle arrest and apoptosis in GBC cell lines. Based on our findings, siRNA or shRNA against UHRF1 therapy may be applied to the treatment of GBC patients. However, due to rapid degradation by serum nucleases, siRNA is very unstable in blood. Moreover, due to its small molecular weight, siRNA is excreted from the body quite rapidly. Establishment of good delivery platforms to deliver siRNA to a targeted location remains the biggest hurdle to overcome prior to using siRNA as a therapeutic strategy and has become an active research area. In recent years, notable advances have been made for the delivery of siRNA to tumors and several promising siRNA delivery platforms have been investigated in order to carry the siRNA into target cells, including liposomes, polyplexes, liposome-polycation-DNA (LPD) and siRNA conjugates (25). In addition, chemical modifications and alternative backbones were used to improve the stability of siRNAs (26). It seems that the need for efficient delivery platforms and stable siRNAs may be met in the near future, and siRNA therapy will provide considerable benefits to cancer patients, including GBC patients.

\section{Acknowledgements}

This study was supported by the National Natural Science Foundation of China (nos. 81272747 and 81372642).

\section{References}

1. Nagorney DM and McPherson GA: Carcinoma of the gallbladder and extrahepatic bile ducts. Semin Oncol 15: 106-115, 1988.

2. Zhu AX, Hong TS, Hezel AF and Kooby DA: Current management of gallbladder carcinoma. Oncologist 15: 168-181, 2010.

3. Takahashi T, Shivapurkar N, Riquelme E, et al: Aberrant promoter hypermethylation of multiple genes in gallbladder carcinoma and chronic cholecystitis. Clin Cancer Res 10: 6126-6133, 2004.

4. Unoki M, Nishidate T and Nakamura Y: ICBP90, an E2F-1 target, recruits $\mathrm{HDAC} 1$ and binds to methyl-CpG through its SRA domain. Oncogene 23: 7601-7610, 2004. 
5. Jeanblanc M, Mousli M, Hopfner R, et al: The retinoblastoma gene and its product are targeted by ICBP90: a key mechanism in the G1/S transition during the cell cycle. Oncogene 24: $7337-7345,2005$

6. Geng Y, Gao Y, Ju H and Yan F: Diagnostic and prognostic value of plasma and tissue ubiquitin-like, containing PHD and RING finger domains 1 in breast cancer patients. Cancer Sci 104: 194-199, 2013

7. Wang F, Zhang P, Ma Y, et al: NIRF is frequently upregulated in colorectal cancer and its oncogenicity can be suppressed by let-7a microRNA. Cancer Lett 314: 223-231, 2012.

8. Unoki M, Kelly JD, Neal DE, Ponder BA, Nakamura Y and Hamamoto R: UHRF1 is a novel molecular marker for diagnosis and the prognosis of bladder cancer. Br J Cancer 101: 98-105 2009.

9. Unoki M, Daigo Y, Koinuma J, Tsuchiya E, Hamamoto R and Nakamura Y: UHRF1 is a novel diagnostic marker of lung cancer. Br J Cancer 103: 217-222, 2010.

10. Babbio F, Pistore C, Curti L, et al: The SRA protein UHRF1 promotes epigenetic crosstalks and is involved in prostate cancer progression. Oncogene 31: 4878-4887, 2012.

11. Yang C, Wang Y, Zhang F, et al: Inhibiting UHRF1 expression enhances radiosensitivity in human esophageal squamous cell carcinoma. Mol Biol Rep 40: 5225-5235, 2013.

12. Pi JT, Lin Y, Quan Q, Chen LL, Jiang LZ, Chi W and Chen HY: Overexpression of UHRF1 is significantly associated with poor prognosis in laryngeal squamous cell carcinoma. Med Oncol 30 $613,2013$.

13. Tien AL, Senbanerjee S, Kulkarni A, et al: UHRF1 depletion causes a $\mathrm{G}_{2} / \mathrm{M}$ arrest, activation of DNA damage response and apoptosis. Biochem J 435: 175-185, 2011.

14. Arima Y, Hirota T, Bronner C, et al: Down-regulation of nuclear protein ICBP90 by p53/p21 $1^{\mathrm{Cip} 1 / \mathrm{WAF} 1}$-dependent DNA damage checkpoint signals contributes to cell cycle arrest at G1/S transition. Genes Cells 9: 131-142, 2004.

15. Lazcano-Ponce EC, Miquel JF, Muñoz N, et al: Epidemiology and molecular pathology of gallbladder cancer. CA Cancer J Clin 51: 349-364, 2001.
16. Jenkins Y, Markovtsov V, Lang W, et al: Critical role of the ubiquitin ligase activity of UHRF1, a nuclear RING finger protein, in tumor cell growth. Mol Biol Cell 16: 5621-5629, 2005.

17. Porter AG and Jänicke RU: Emerging roles of caspase-3 in apoptosis. Cell Death Differ 6: 99-104, 1999.

18. Vermeulen K, Van Bockstaele DR and Berneman ZN: Apoptosis: mechanisms and relevance in cancer. Ann Hematol 84: 627-639, 2005.

19. Guan D, Factor D, Liu Y, Wang Z and Kao HY: The epigenetic regulator UHRF1 promotes ubiquitination-mediated degradation of the tumor-suppressor protein promyelocytic leukemia protein. Oncogene 32: 3819-3828, 2013.

20. Chang HJ, Yoo BC, Kim SW, Lee BL and Kim WH: Significance of PML and p53 protein as molecular prognostic markers of gallbladder carcinomas. Pathol Oncol Res 13: 326-335, 2007.

21. Krieghoff-Henning E and Hofmann TG: Role of nuclear bodies in apoptosis signalling. Biochim Biophys Acta 1783: 2185-2194, 2008.

22. Reineke EL, Liu Y and Kao HY: Promyelocytic leukemia protein controls cell migration in response to hydrogen peroxide and insulin-like growth factor-1. J Biol Chem 285: 9485-9492, 2010.

23. Kim WH, Kang KH, Kim MY and Choi KH: Induction of p53-independent p21 during ceramide-induced G1 arrest in human hepatocarcinoma cells. Biochem Cell Biol 78: 127-135, 2000.

24. Zuo S, Liu C, Wang J, et al: IGFBP-rP1 induces p21 expression through a p53-independent pathway, leading to cellular senescence of MCF-7 breast cancer cells. J Cancer Res Clin Oncol 138: 1045-1055, 2012.

25. Shen Y: Advances in the development of siRNA-based therapeutics for cancer. IDrugs 11: 572-578, 2008.

26. Unoki M, Kumamoto K and Harris CC: ING proteins as potential anticancer drug targets. Curr Drug Targets 10: 442-454, 2009. 